\title{
PARP and Cancer — If It's Broke, Don't Fix It
}

\author{
Lisa A. Carey, M.D. and Norman E. Sharpless, M.D. \\ Departments of Medicine and Genetics, the Line-berger Comprehensive Cancer Center, \\ University of North Carolina School of Medicine, Chapel Hill
}

\begin{abstract}
With apologies to Nietzsche, who once said "that which does not kill us makes us stronger," sometimes that which does not kill weakens us so the next blow will be lethal. In this issue of the Journal, O'Shaughnessy and colleagues ${ }^{1}$ describe a study in which poly(adenosine diphosphate-ribose) polymerase (PARP) inhibition was added to cytotoxic chemotherapy in an effort to set breast cancers up for that "next blow" (ClinicalTrials.gov number, NCT00540358). PARP inhibitors represent an exciting new therapeutic direction in oncology: the rational targeting of tumor-cell vulnerability during DNA repair.
\end{abstract}

Cellular DNA is under constant assault; over 150 genes, in multiple pathways, sense and repair damaged DNA. Response to DNA damage is complex and interconnected but contains several potential drug targets, including PARPs. Repair of DNA double-strand breaks is particularly interesting, since such breaks are the death-provoking effectors of therapeutic radiation and some cytotoxic chemotherapy. A favored mechanism for repair of double-strand breaks is homologous recombination, a BRCA1/2-dependent, high-fidelity process in which the homologous sequence is used to precisely repair the break. A patient with an inherited $B R C A 1$ or $B R C A 2$ mutation has normal BRCA function, owing to the one functional allele, but in the patient's cancer that allele is generally inactivated, rendering the tumor cells deficient in homologous recombination. PARP inhibitors exploit this tumorspecific defect in repairing double-strand breaks.

The PARPs, the most abundant of which is PARP1, are a family of nuclear enzymes that polymerize poly(adenosine diphosphate-ribose) on substrate proteins to regulate processes such as DNA repair, gene transcription, and chromatin architecture. ${ }^{2}$ PARP1 is thought to play a role in the repair of single-strand breaks, which can become toxic double-strand breaks. In cells with intact homologous recombination, double-strand breaks that occur as a result of PARP inhibition can be resolved, but in tumor cells lacking homologous recombination, PARP inhibition leads to persistent double-strand breaks, inducing cell death. ${ }^{3}$ Inhibition of homologous recombination or PARPs may be well tolerated in isolation, but combined inactivation of these distinct DNA-repair pathways results in cell death - a process called "synthetic lethality."

Initial data on PARP inhibitors came from a phase 1 trial $^{4}$ of patients with breast, ovarian, or prostate cancer who had been extensively treated previously. The patients received singleagent therapy with olaparib; there was significant tumor reduction only in patients with a germline $B R C A 1$ or $B R C A 2$ mutation. Further evidence of the efficacy of PARP inhibitors came from a phase 2 study ${ }^{5}$ limited to patients with a germline $B R C A 1$ or $B R C A 2$ mutation and with advanced breast cancer, among whom $41 \%$ had a response to olaparib alone at the recommended phase 2 dose.

Copyright () 2011 Massachusetts Medical Society. All rights reserved

Disclosure forms provided by the authors are available with the full text of this article at NEJM.org. 
BRCA1/2-associated cancers are rare, representing less than $5 \%$ of breast-cancer cases, so it is important to find out whether PARP inhibitors have activity in other cancers. A likely candidate is triple-negative breast cancer; these tumors lack expression of the estrogen receptor (ER), progesterone receptor (PR), and human epidermal growth factor receptor 2 (HER2). ${ }^{6}$ No biologic or targeted therapy is known to have efficacy in triple-negative breast cancer, and the prognosis is poor after relapse. Most triple-negative breast cancers are of a molecular subtype called basal-like, as are the majority of $B R C A 1$-associated breast cancers. $B R C A 1$-associated and sporadic basal-like breast cancers share certain biologic characteristics. This notion of shared "BRCAness" is the rationale for testing PARP inhibition in sporadic triple-negative breast cancer, since even without germline BRCA1 mutations, these tumors may harbor other lesions that diminish homologous recombination.

In the randomized, phase 2 trial by O'Shaughnessy and colleagues, patients with metastatic triple-negative breast cancer received the chemotherapy doublet gemcitabine-carboplatin either alone or in combination with the PARP inhibitor iniparib. The rate of clinical benefit, a measure of durable response or disease stabilization, was 56\% in the iniparib group, as compared with $34 \%$ in the chemotherapy-alone group. There was negligible additional toxicity with iniparib. These findings alone would catch one's attention, but the improvement in progression-free survival by 2 months and improvement in overall survival by nearly 5 months with iniparib make this an even more compelling story.

Both excitement and caution are appropriate in interpreting the trial by O'Shaughnessy and colleagues. Some clear drawbacks should be noted. The cohort was small, the end points were assessed by the investigators, the gemcitabine-carboplatin regimen is unconventional, and there were imbalances at baseline in prognostically important characteristics favoring the iniparib group. We cannot tell whether the benefit from the PARP inhibitor accrued to all triple-negative tumors equally or whether the benefit preferentially accrued to a subgroup of BRCA-deficient tumors, with less effect in those without the deficiency.

Even if iniparib should reproducibly demonstrate activity in triple-negative breast cancer, questions remain about the compound used in this study. In small studies, other PARP inhibitors have not shown promising results outside of BRCA-associated breast cancer. ${ }^{7,8}$ In addition, unlike with iniparib, it has been challenging to combine several of these other agents with chemotherapy. Iniparib is a much less potent inhibitor of PARP1 (with approximately $0.1 \%$ the potency) than most other agents of this class. ${ }^{9}$ The present study does not include a pharmaco-dynamic assessment of PARP activity in the patients receiving iniparib, and it is unclear whether the therapeutic efficacy of this agent correlates with PARP inhibition in these patients. Therefore, the low potency, reduced toxicity when combined with chemotherapy, and possible BRCA-independent activity of iniparib distinguish it from other members of the class; at least part of its antitumor efficacy may be independent of PARP inhibition.

O'Shaughnessy and colleagues are conducting a phase 3 trial of iniparib (NCT00938652). If the phase 2 results reported here are confirmed in the larger study, PARP inhibition could be a rational approach to treating triple-negative breast cancer, and the first therapy showing a survival advantage over chemotherapy alone - but important questions would remain. First, does the activity of iniparib in this trial result from PARP inhibition or an unknown mechanism? More generally, since the "BRCAness" of triple-negative breast cancers is not proved, do PARP inhibitors as a class have activity in cancers lacking BRCA1 or BRCA2 dysfunction? Can PARP inhibition augment DNA-damaging chemotherapy administered to other subtypes of breast cancer or other types of tumor? Which DNA-damaging chemotherapy best synergizes with PARP inhibitors? Adjuvant trials of PARP inhibitors for patients with early-stage breast cancer, in which these drugs will be added to chemotherapy 
delivered with a curative intent, are already being developed. The many roles of PARPs outside of DNA repair raise concern that PARP inhibitors may exhibit as-yet unknown "ontarget" toxic effects, such as the diet-induced obesity and insulin resistance seen in PARP1deficient mouse models. ${ }^{10}$ In addition, the risk of secondary cancer from DNA-repair inhibition needs to be considered carefully if these agents are used for longer periods in healthier patients.

Caveats notwithstanding, these are exciting results presaging improved therapy for an underserved subgroup of patients with breast cancer and, we hope, heralding a new approach of "setting cancers up for the next blow" by combining cytotoxic chemotherapy with agents directly targeting the DNA-damage response.

\section{References}

1. O'Shaughnessy J, Osborne C, Pippen JE, et al. Iniparib plus chemotherapy in metastatic triplenegative breast cancer. N Engl J Med. 2011; 364:205-14. [PubMed: 21208101]

2. Krishnakumar R, Kraus WL. The PARP side of the nucleus: molecular actions, physiological outcomes, and clinical targets. Mol Cell. 2010; 39:8-24. [PubMed: 20603072]

3. Farmer H, McCabe N, Lord CJ, et al. Targeting the DNA repair defect in BRCA mutant cells as a therapeutic strategy. Nature. 2005; 434:917-21. [PubMed: 15829967]

4. Fong PC, Boss DS, Yap TA, et al. Inhibition of poly(ADP-ribose) polymerase in tumors from BRCA mutation carriers. N Engl J Med. 2009; 361:123-34. [PubMed: 19553641]

5. Tutt A, Robson M, Garber JE, et al. Oral poly(ADP-ribose) polymerase inhibitor olaparib in patients with BRCA1 or BRCA2 mutations and advanced breast cancer: a proof-of-concept trial. Lancet. 2010; 376:235-44. [PubMed: 20609467]

6. Foulkes WD, Smith IE, Reis-Filho JS. Triple-negative breast cancer. N Engl J Med. 2010; 363:1938-48. [PubMed: 21067385]

7. Gelmon KA, Hirte HW, Robidoux A, et al. Can we define tumors that will respond to PARP inhibitors? A phase II correlative study of olaparib in advanced serous ovarian cancer and triplenegative breast cancer. J Clin Oncol. 2010; 28:3002. abstract. http://www.asco.org/ASCOv2/ Meetings/Abstracts?\&vmview=abst_detail_view\&confID=74\&abstractID=50240 [PubMed: 20479400]

8. Isakoff SJ, Overmoyer B, Tung NM, et al. A phase II trial of the PARP inhibitor veliparib (ABT888) and temozolomide for metastatic breast cancer. J Clin Oncol. 2010; 28:1019. abstract. http://www.asco.org/ascov2/Meetings/Abstracts? \&vmview=abst_detail_view\&confID=74\&abstractID=43191

9. He JX, Yang CH, Miao ZH. Poly(ADP-ribose) polymerase inhibitors as promising cancer therapeutics. Acta Pharmacol Sin. 2010; 31:1172-80. [PubMed: 20676117]

10. Devalaraja-Narashimha K, Padanilam BJ. PARP1 deficiency exacerbates diet-induced obesity in mice. J Endocrinol. 2010; 205:243-52. [PubMed: 20338998] 